\title{
The evolutionary history of $D$. buzzatii. XXII. Chromosomal and genic sterility in male hybrids of Drosophila buzzatii and Drosophila koepferae
}

\author{
H. NAVEIRA \& A. FONTDEVILA \\ Departamento de Genética y Microbiologí, Universidad Autónoma de Barcelona, Bellaterra (Barcelona), Spain
}

\begin{abstract}
The genetic basis of sterility in $\mathrm{F}_{1}$ male hybrids of Drosophila buzzatii and D. koepferae has been investigated in two steps. (1) By successive backcrossing of hybrid females to either parental species. (2) By assessment of the effects on male fertility of selected segments of polytene chromosomes from the donor species on a background entirely derived from the recipient species. The length of introgressed segments producing sterility was progressively reduced through repeated backcrosses. This procedure sometimes led to an approximate mapping of major genes of hybrid sterility (genic sterility) on the polytene chromosome map. At other times it was found that sterility was produced only when the introgressed segment exceeded a certain threshold size (chromosomal sterility). The contribution of the autosomes to hybrid sterility seems to be mainly of the chromosomal type. The evidence concerning the $\mathbf{X}$ chromosome is equivocal. No fertile males were found following introgression with any of the investigated segments of this chromosome. These results are compatible both with the presence of at least six major genes of hybrid sterility (genic sterility) and with the existence of a rather small threshold size for the chromosome segments producing sterility (chromosomal sterility). The role of the Y chromosome was not investigated in this study.
\end{abstract}

Keywords: Drosophila, hybrid male sterility, major genes, polygenes.

\section{Introduction}

The genetic causes of intraspecific male sterility in Drosophila have been gathered into two groups (Lifschytz \& Lindsley, 1974; Lindsley \& Tokuyasu, 1980): genic (specific) and chromosomal (non-specific). Genic sterility results from the mutation of specific genes whose products are necessary (directly or indirectly) at one or other stage for a normal spermatogenesis. Chromosomal sterility, on the other hand, cannot be attributed to the effects of particular gene loci (non-specific), but results from an abnormal chromosome organization, with a strict dependence on the size of the chromosome segments involved. Genes responsible for intraspecific genic sterility show an easily recognizable, clear-cut segregation, and are generally recessive. They are spread all over the Drosophila genome, including the $\mathrm{Y}$ chromosome, their

Correspondence: Dr H. Naveira, Departamento de Genética y Microbiología, Facultad de Ciencias, Universidad Autónoma de Barcelona, Bellaterra (Barcelona), Spain. distribution is roughly proportional to the relative length of each chromosome, and their total number is estimated at about 500 (Lindsley \& Tokuyasu, 1980). On the contrary, the genetic basis of chromosomal sterility remains largely unknown, although its relationship with chromosome size strongly suggests the involvement of polygenes. It is exhibited by the socalled chromosomal mutants of $D$. melanogaster, which comprise reciprocal and insertional $\mathrm{X}$-autosome translocations. However, it has nothing to do with segregational problems at meiosis, and is most probably brought about by an interference with the asynchronous inactivation of the $\mathrm{X}$ and the autosomes in the primary spermatocytes (Lindsley \& Tokuyasu, 1980). A remarkable feature of these mutants is that the sterility depends on the size of the translocated chromosome segment. When the size of the segment (either from the $\mathrm{X}$, the autosome, or both at the same time) is roughly greater than 50 per cent of a chromosome arm in D. melanogaster, sterility ensues (Lindsley \& Lifschytz, 1972). 
Most studies on interspecific hybrid male sterility have only paid attention to genic sterility, ignoring the possible existence of chromosomal sterility. To date, there is only one report on 'chromosomal sterility' in hybrid males (Naveira \& Fontdevila, 1986): in the hybrids of $D$. buzzatii and $D$. koepferae, formerly $D$. serido from Argentina (Fontdevila et al., 1988). In this case the sterilizing effect of the autosomes is apparently caused by the introgression of more than a minimum number of polygenes, each contributing to a very small, individually undetectable, effect on the disruption of hybrid spermatogenesis (Naveira et al. 1984, 1989; H. Naveira \& A. Fontdevila, unpublished manuscript). The characteristics of this type of hybrid male sterility agree with the said properties of chromosomal sterility in X-autosome translocations of $D$. melanogaster. It is dominant, not attributable to particular gene loci (nonspecific), and shows a remarkable dependence on the size of the introgressed chromosome segments. In both cases, sets of genes that are known to function normally in situ are placed in new genetic environments in which their expression is suboptimal; the cumulative effects of doing this to a sufficient number of genes leads to male infertility. Only one specimen of each chromosome of the haploid complement was examined in our former report (Naveira \& Fontdevila, 1986). We have now undertaken a systematic study of several chromosome specimens in order to assess the relative importance of genic and chromosomal sterility in these interspecific hybrids.

\section{Materials and methods}

By means of a new method of cytogenetic localization (Naveira et al., 1986), we have investigated the genetic basis of sterility in male hybrids of $D$. buzzatii and $D$. koepferae. Eight independent strains of D. koepferae were used as donor species for the introgression into $D$. buzzatii. On the other hand, only one strain of $D$. buzzatii (strain b0) could successfully be used as a donor. This same $D$. buzzatii strain was used as a recipient species, together with another seven strains. All hybridizations involved one strain of D. koepferae and one strain of $D$. buzzatii, and therefore can be considered as independent samples of the interaction of the genomes of both species. Laboratory crosses between $D$. koepferae females and $D$. buzzatii males yield an $F_{1}$ of sterile hybrid males and fertile hybrid females. $F_{1}$ hybrid females were backcrossed to each parental species, and this backcrossing was continued for several generations, giving rise to different introgression lines where the effecct of different chromosome segments on hybrid male fertility could be tested (Naveira \& Fontdevila, 1986). The rationale of this method is to reduce progressively, through repeated backcrosses and the accompanying recombination, the length of introgressed segments containing major dominant sterility genes. Asynapsis of homologous polytene chromosomes of $D$. buzzatii and $D$. koepferae was used to identify the segments introgressed in each line. The validity of this method for mapping gene differences between species has been already demonstrated (Naveira et al., 1986) and recently ratified by in-situ hybridizations (Labrador et al., 1990). All technical details concerning the experimental cultures of Drosophila, the crossing design for introgressive hybridization, and the preparation of polytene chromosomes from salivary glands and Malpighian tubes are given elsewhere (Naveira et al., 1986; Naveira \& Fontdevila, 1986). References to cytological subdivisions of the polytene chromosomes of $D$. buzzatii and $D$. koepferae correspond to commonly used descriptions in the repleta group (Wharton, 1942; Ruiz et al., 1982; see also Fig. 1 in this paper).

\section{Origin of the strains}

All the strains used in this work were derived from flies collected in December 1979 by A. Fontdevila and A. Ruiz in Argentina (see Fontdevila et al., 1982, for a description of collection sites). D. buzzatii strains were derived either directly from the $F_{1}$ progeny of single, wild, inseminated females (isolines) collected at the Sierra de San Luis, or from a couple of flies (male and virgin female) taken from a population cage founded by the combination of 138 isolines from the same collection. All strains were kept by mass culturing thereafter, except for strain b0, which was kept by brother-sister matings during several generations prior to mass matings. Another strain, bX, was fixed for a new inversion on chromosome $5(\operatorname{In}(5) 3)$ produced in our laboratory, which permitted the introgression of the cytological interval 5D1-5F2 (Table 1). Four D. koepferae strains were derived from single inseminated females taken from a population cage founded by the combination of 59 isolines also collected at Sierra de San Luis. Another four strains were derived directly from single isolines collected at Vipos. All the strains were kept by mass culturing.

\section{Sterility tests}

Thirty offspring males, 6-7 days old, from each hybrid female, were placed individually with two $D$. buzzatii virgin females in small food vials. The cultures were examined 8 days later. Five to 10 of the males from cultures without larvae were dissected, their seminal vesicles were checked for the presence of sperm, and the polytene chromosomes of their Malpighian tubes 
were analysed. The rest of the flies were transferred to fresh food vials every week until their death. Each one of the 30 males was finally identified as fertile or sterile, according to the presence or absence of progeny in the vials. Whenever a male produced progeny, the polytene chromosomes of eight larvae were analysed, and the male karyotype was inferred from these data.

Controls of these sterility tests were performed by analysing the progeny of non-hybrid sisters of the selected hybrid females. At least two replicates were run of each introgressed segment, thus making a total of 60 males examined. An introgressed segment is considered to produce sterility when not one of the 60 males is a fertile hybrid.

\section{Results}

Each cytological subdivision of the chromosome maps of both $D$. koepferae and $D$. buzzatii were analysed in several independent chromosome specimens for the presence of major sterility genes (Table 1). None of the subdivisions of the autosomes of $D$. koepferae were found to contain major dominant sterility genes. Hybrid sterility was always produced by the introgression of relatively large chromosome segments from $D$. koepferae into $D$. buzzatii. As illustrated in Fig. 1a for chromosome 3 , a set of overlapping segments covering the whole of chromosomes $3,4,5$ and 6 was obtained from fertile hybrid males, and we may therefore conclude that no single gene that could produce sterility by itself was present in the specimens of chromosomes analysed. On the other hand, only one specimen of autosomes 3 and 4 of $D$. buzzatii (strain b0) could be successfully introgressed into D. koepferae. Among the cytological subdivisions of these chromosomes, only one was found to contain a major dominant sterility gene, which was mapped in the interval E1e-E1h (four polytene bands) of chromosome 3, as shown in Fig. 1 b. Heterozygosis with $D$. koepferae in this region, however, is not a sufficient condition for hybrid male sterility. There must be some kind of interaction with the genetic background (perhaps with the Y chromosome), because the reciprocal introgression $(3 \mathrm{E} 1 \mathrm{e}-\mathrm{E} 1 \mathrm{~h}$ of $D$. koepferae into $D$. buzzatii b0) allows fertility (Fig. 1a). In addition, hybrid sterility was also produced by introgression in excess of a threshold chromosome size. As an estimation of this threshold, we take the size half-way between the maximum for fertility and the minimum for sterility (Table 2). The maximum for fertility is the largest introgressed segment found in fertile hybrid males, whereas the minimum for sterility is the smallest introgressed segment found in sterile hybrid males. Apparently this threshold is not
Table 1 Number of independent specimens of chromosomes from $D$. koepferae analysed for the presence of major dominant genes of hybrid sterility in each of the different cytological subdivisions

\begin{tabular}{|c|c|}
\hline $\begin{array}{l}\text { Cytological } \\
\text { subdivisions }\end{array}$ & $\begin{array}{l}\text { Number of specimens } \\
\text { of chromosome }\end{array}$ \\
\hline \multicolumn{2}{|l|}{ Chromosome $1(\mathrm{X})$ : } \\
\hline From $1 \mathrm{~A} 1$ to $1 \mathrm{H}$ & 4 \\
\hline \multicolumn{2}{|l|}{ Chromosome 2: } \\
\hline From $2 \mathrm{~A} 1$ to $2 \mathrm{~B} 2$ & 5 \\
\hline From $2 \mathrm{~B} 1$ to $2 \mathrm{~A} 3$ & 4 \\
\hline $2 \mathrm{Cl}$ & 3 \\
\hline From $2 \mathrm{C} 2$ to $2 \mathrm{C} 7$ & 1 \\
\hline From $2 \mathrm{C} 6$ to $2 \mathrm{~F} 4$ & 0 \\
\hline $2 F 3$ & 3 \\
\hline $2 \mathrm{G} 1$ & 4 \\
\hline From $2 \mathrm{G} 2$ to $2 \mathrm{H}$ & 5 \\
\hline \multicolumn{2}{|l|}{ Chromosome 3: } \\
\hline From $3 \mathrm{~A} 1$ to $3 \mathrm{C} 4$ & 6 \\
\hline $3 \mathrm{C} 5$ & 4 \\
\hline From $3 \mathrm{D} 1$ to $3 \mathrm{~F} 4$ & 3 \\
\hline 3G1 & 5 \\
\hline From $3 \mathrm{G} 2$ to $3 \mathrm{H}$ & 6 \\
\hline \multicolumn{2}{|l|}{ Chromosome 4: } \\
\hline From $4 \mathrm{~A} 1$ to $4 \mathrm{~B} 3$ & 5 \\
\hline From $4 \mathrm{~B} 4$ to $4 \mathrm{C} 1$ & 4 \\
\hline $4 \mathrm{C} 2$ & 5 \\
\hline From 4C3 to 4D4 & 6 \\
\hline From $4 \mathrm{D} 5$ to $4 \mathrm{E} 1$ & 5 \\
\hline From $4 \mathrm{E} 2$ to $4 \mathrm{G} 2$ & 3 \\
\hline From $4 \mathrm{G} 3$ to $4 \mathrm{H}$ & 5 \\
\hline \multicolumn{2}{|l|}{ Chromosome 5: } \\
\hline From $5 \mathrm{~A} 1$ to $5 \mathrm{C} 3$ & 6 \\
\hline $5 \mathrm{C} 4$ & 3 \\
\hline $5 \mathrm{C} 5$ & 2 \\
\hline From $5 \mathrm{D} 1$ to $5 \mathrm{~F} 2$ & 1 \\
\hline $5 F 3$ & 3 \\
\hline $5 \mathrm{G} 1$ & 5 \\
\hline From $5 \mathrm{G} 2$ to $5 \mathrm{H}$ & 6 \\
\hline \multicolumn{2}{|l|}{ Chromosome 6 (dot): } \\
\hline From $6 \mathrm{~A} 1$ to $6 \mathrm{H}$ & 5 \\
\hline
\end{tabular}

Intervals 2C2-2F 4 and 5D1-5F2 are within chromosome inversions fixed in either species, and their analysis was only possible when double recombinants could be obtained.

the same for all chromosomes or chromosome regions. To facilitate comparisons we recognized three chromosomal regions: telomeric (or distal, including the telomere), central (including the central cytological subdivision D5) and centromeric (or proximal, including the centromere). Data for chromosome 2, and central and centromeric regions of chromosome 5 could not be obtained because of 

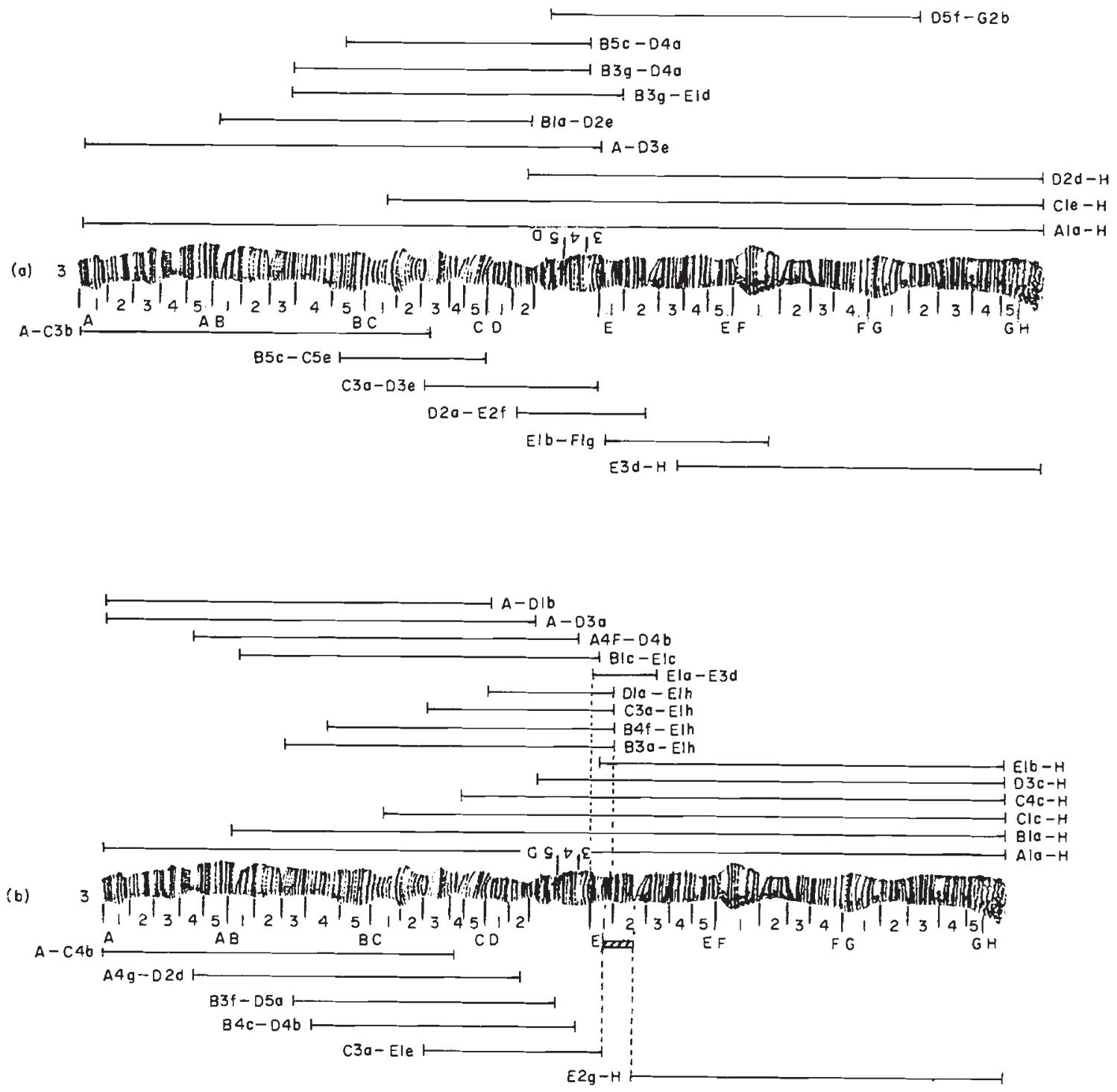

Fig. 1 (a) Introgression of chromosome 3 segments from D. koepferae into D. buzzatii (strain b0). (b) Reciprocal (strain b0) into D. koepferae. Segments depicted above the chromosome produce hybrid male sterility. Segments depicted below the chromosome allow fertility. Each segment was separately introgressed.

fixed inversions in either species, which set bounds to the sizes of the segments produced by crossing over. The threshold size is smaller in the central region than in the extremes of chromosome 3 , smaller in chromosome 4 than in chromosomes 3 or 5 , and probably smaller for $D$. koepferae than for $D$. buzzatii. All this evidence points to a certain degree of heterogeneity in the distribution along the chromosomes of the genetic factors (polygenes) responsible for the size effect on fertility. The threshold size comprises between 5 and 9 per cent of the haploid polytene karyotype in $D$. koepferae, and between 6 and 9 per cent in D. buzzatii. This amounts to an introgression of between one-third and one-quarter of the length of the corresponding chromosome. With regard to the $X$ chromosome, its contribution to hybrid male sterility must be very important. None of the $\mathrm{X}$ chromosome segments from D. koepferae introgressed into $D$. buzzatii allowed male fertility. Our analysis points either to at least six major genes of hybrid sterility (genic sterility) or to a very small threshold size for the chromosome segments producing sterility (chromosomal sterility). We have been able so far to introgress only six non-overlapping segments of the $\mathrm{X}$ chromosome from $D$. koepferae into D. buzzatii: A-A2a (0.9 per cent of the haploid 
Table 2 Average maximum size of the introgressed segments in fertile hybrids and average minimum size in sterile hybrids, for chromosomes 3,4 , and 5 of $D$. koepferae and D. buzzatii. Size is expressed as a percentage of the total length of the haploid polytene karyotype. Averages and standard errors (in parentheses) are calculated over the independent specimens analysed in each chromosome region

\begin{tabular}{|c|c|c|c|c|}
\hline \multirow[b]{2}{*}{$\begin{array}{l}\text { Chromosome } \\
\text { regions }\end{array}$} & \multicolumn{2}{|l|}{ D. koepferae } & \multicolumn{2}{|l|}{ D. buzzatii } \\
\hline & $\begin{array}{l}\text { Maxima for } \\
\text { fertility }\end{array}$ & $\begin{array}{l}\text { Minima for } \\
\text { sterility }\end{array}$ & $\begin{array}{l}\text { Maxima for } \\
\text { fertility }\end{array}$ & $\begin{array}{l}\text { Minima for } \\
\text { sterility }\end{array}$ \\
\hline \multicolumn{5}{|l|}{ Chromosome 3: } \\
\hline Telomeric & $7.1(0.27)$ & $10.2(0.69)$ & 8.3 & 9.0 \\
\hline Central & $4.2(0.18)$ & $6.8(1.39)$ & 6.1 & 8.5 \\
\hline Centromeric & $7.3(0.58)$ & $11.1(1.02)$ & 8.6 & - \\
\hline \multicolumn{5}{|l|}{ Chromosome 4: } \\
\hline Telomeric & $4.0(0.24)$ & $7.5(0.17)$ & - & - \\
\hline Central & $4.6(0.27)$ & $7.9(0.52)$ & 5.9 & 6.9 \\
\hline Centromeric & $5.1(0.09)$ & $7.4(0.38)$ & 6.3 & 7.8 \\
\hline $\begin{array}{c}\text { Chromosome } 5: \\
\text { Telomeric }\end{array}$ & $6.3(0.29)$ & $8.5(0.23)$ & - & - \\
\hline
\end{tabular}

- Data unavailable.

polytene karyotype), B2b-B4f (1.2 per cent), C1b-C 4a (1.6 per cent), D 3a-D 3e (0.3 per cent), $\mathrm{E} 3 \mathrm{~d}-\mathrm{E} 4 \mathrm{~g}$ (0.9 per cent), and $\mathrm{F} 4 \mathrm{~d}-\mathrm{H}$ (3.6 per cent). Any of these segments are sufficient to cause sterility.

\section{Discussion}

We have found that the contribution of the autosomes to hybrid male sterility seems to be mainly of the chromosomal type, while the evidence concerning the $\mathrm{X}$ chromosome is still equivocal. No fertile male was found following introgression with any of the investigated segments of the $\mathrm{X}$ chromosome, but the results are compatible both with the presence of at least six major genes of hybrid sterility (genic sterility) and with the existence of a rather small threshold size for the chromosome segments producing sterility (chromosomal sterility). The $\mathrm{Y}$ chromosome could not be investigated in these experiments $\left(F_{1}\right.$ hybrid males are always sterile).

Hybrid male sterility (or inviability) is very often the only manifestation of postzygotic reproductive isolation between species of Drosophila, but apparently it is only the initial phase of a single evolutionary pathway that culminates in the sterility (or inviability) of both sexes (Coyne \& Orr, 1989): an initial accumulation of alleles causing sterility in male hybrids is followed by the accumulation of alleles causing sterility in female hybrids. The identification of these alleles, but particu- larly of those specific to males, has therefore attracted much attention because they constitute an essential part of the differentiation process that leads to speciation.

It is known that certain $\mathrm{Y}$ chromosome-autosome recessive interactions bring about sterility in male hybrids of different Drosophila species (Stone, 1947; Alexander et al., 1952; Hennig, 1977; Schäfer, 1978; Vigneault \& Zouros, 1986; Pantazidis \& Zouros, 1988; Zouros et al., 1988) but in this paper we are more interested in dominant mutations: those that cause sterility in heterozygosis with the other species alleles, as happens in $F_{1}$ males. Except for cne case (Pontecorvo, 1943), where interacting polygenic sets were assumed to cause hybrid male sterility, the evidence from hybrids of different species has always been interpreted as evidence for the existence of a limited number of major segregating units, or 'sterility genes' (Dobzhansky, 1936; Coyne, 1984; Coyne \& Kreitman, 1986; Orr, 1987; 1989a,b; Coyne \& Charlesworth, 1989; Orr \& Coyne, 1989). Each of these genes was assumed to cause sterility (genic sterility) by itself, thereby ignoring alternative interpretations based on other possible genetic causes of male sterility (chromosomal sterility). In fact, most of this evidence, perhaps with the exception of Orr (1989b), is not conclusive, because the results were often derived from the introgression of chromosome segments of unknown size. In addition, the possible existence of 
some major genes of hybrid sterility is not really the point of this debate. Instead, it is their relative importance when compared with minor genes in the evolution of this isolating barrier. Progressively more differentiated species could either show higher numbers of major sterility genes, expressed in hybrids, or higher numbers of polygenes, which would be manifest as a progressive reduction in threshold chromosome sizes for hybrid sterility. The distinction between genic sterility (major genes) and chromosomal sterility (polygenes) is an essential one if we want to arrive at an understanding of the speciation process in Drosophila, because their modes of evolution may be quite different. According to the results of this paper, chromosomal causes of hybrid sterility might be the rule rather than the exception. New experiments should be designed to assess the relative importance of these two types of factors in other interspecific hybrids and to unveil their mode of function.

\section{Acknowledgements}

This work was supported by grant $0910 / 81$, awarded to A.F. by the 'Comisión Asesora de Investigación Científica y Técnica', and a research scholarship awarded to H.N. from the 'Programa de Formación de Personal Investigador' (Spain). We thank B. Charlesworth and D. L. Lindsley for their useful comments on this manuscript.

\section{References}

ALEXANDER, M. L., LEA, R. B. AND STONE, w. s. 1952. Interspecific gene variability in the virilis group. Univ. Texas Publ., 5204, 106-113.

COYNE, J. A. 1984. Genetic basis of male sterility in hybrids between two closely related species of Drosophila. Proc. Natl. Acad. Sci., USA, 81, 4444-4447.

COYNE, J. A. AND CHARLESWORTH, B. 1989. Genetic analysis of $\mathrm{X}$-linked sterility in hybrids between three sibling species of Drosophila. Heredity, 62, 97-106.

COYNE, J. A. AND KREITMAN, M. 1986. Evolutionary genetics of two sibling species of Drosophila, D. simulans and $D$. mauritiana. Evolution, 40, 673-691.

COYNE, J. A. AND ORR, H. A. 1989. Patterns of speciation in Drosophila. Evolution, 43, 362-381.

DOBZHANSKY, TH. 1936. Studies on hybrid sterility. II. Localization of sterility factors in Drosophila pseudoobscura hybrids. Genetics, 21, 113-135.

FONTDEVIlA, A., Pla, C., HASson, E. et al. 1988. Drosophila koepferae: a new member of the Drosophila serido (Diptera: Drosophilidae) superspecies taxon. Ann. Entomol. Soc. Am., 81, 380-385.

FONTDEVILA, A., RUIZ, A., OCAÑA, J. AND ALONSO, G. 1982. The evolutionary history of $D$. buzzatii. II. How much has chromosomal polymorphism changed in colonization?
Evolution, 36, 843-851.

HENNIG, w. 1977. Gene interactions in germ cell differentiation of Drosophila. Adv. Enz. Reg., 15, 363-371.

LABRADOR, M., NAVEIRA, H. AND FONTDEVILA, A. 1990. Genetic mapping of the Adh locus in the repleta group of Drosophila by 'in situ' hybridization. J. Hered., 81, 83-86.

LIFSCHYTZ, E. AND LINDSLEY, D. L. 1974. Sex chromosome inactivation during spermatogenesis. Genetics, 78, 323-331.

LINDSLEY, D. L. AND LIFSCHYTZ, E. 1972. The genetic control of spermatogenesis in Drosophila. In: Beatty, R. A. and Glueckson-Waelsch, S. (eds) The Genetics of the Spermatozoon, University of Edinburgh Press, Edinburgh, pp. 203-222.

LINDSLEY, D. L. AND TOKUYASU, K. T. 1980. Spermatogenesis. In: Ashburner, M. and Wright, T. R. F. (eds) The Genetics and Biology of Drosophila, Vol. 2d, Academic Press, New York, pp. 225-294.

NAVEIRA, H. AND FONTDEVILA, A. 1986. The evolutionary history of Drosophila buzzatii. XII. The genetic basis of sterility in hybrids between $D$. buzzatii and its sibling $D$. serido from Argentina. Genetics, 114, 841-857.

NAVEIRA, H., HAUSCHTECK-JUNGEN, E. AND FONTDEVILA, A. 1984. Spermiogenesis of inversion heterozygotes in backcross hybrids between Drosophila buzzatii and D. serido. Genetica, 65, 205-214.

NAVEIRA, H., HAUSCHTECK-JUNGEN, E. AND FONTDEVILA, A. 1989. The evolutionary history of Drosophila buzzatii. XV. Meiosis of inversion heterozygotes in backcross hybrids between $D$. buzzatii and its sibling $D$. koepferae. Genome, 32, 262-270.

NAEIRA, H., PLA, C. AND FONTDEVILA, A. 1986. The evolutionary history of $D$. buzzatii. XI. A new method for cytogenetic localization based on asynapsis of polytene chromosomes in interspecific hybrids of Drosophila. Genetica, 71, 199-212.

ORR, H. A. 1987. Genetics of male and female sterility in hybrids of Drosophila pseudoobscura and D. persimilis. Genetics, 116, 555-563.

ORR, H. A. 1989a. Genetics of sterility in hybrids between two subspecies of Drosophila. Evolution, 43, 180-189.

ORR, H. A. 1989 b. Localization of genes causing postzygotic isolation in two hybridizations involving Drosophila pseudoobscura. Heredity, 63, 231-237.

ORR, H. A. AND COYNE, J. A. 1989. The genetics of postzygotic isolation in the Drosophila virilis group. Genetics, 121, 527-537.

PANTAZIDIS, A. C. AND Zouros, E. 1988. Location of an autosomal factor causing sterility in Drosophila mojavensis males carrying the Drosophila arizonensis Y chromosome. Heredity, 60, 299-304.

PONTECORVO, G. 1943. Hybrid sterility in artificially produced recombinants between Drosophila melanogaster and $D$. simulans. Proc. R. Soc. Edin. Sect. B, 61, 385-397.

RUIZ, A., FONTDEVILA, A. AND WASSERMAN, M. 1982. The evolutionary history of Drosophila buzzatii. III. Cytogenetic relationships between two sibling species of the buzzatii cluster. Genetics, 101, 503-518.

SCHAFER, U. 1978. Sterility in Drosophila hydei $\times D$. neohydei hybrids. Genetica, 49, 205-214. 
STONE, w. S. 1947. Gene replacement in the virilis group. Univ. Texas Publ., 4720, 161-166.

VIGNEAULT, G. AND zouros, E. 1986. The genetics of asymmetrical male sterility in Drosophila mojavensis and Drosophila arizonensis hybrids: Interactions between Y-chromosome and autosomes. Evolution, 40, 1160-1170.
Wharton, L. 1942. Analysis of the repleta group of Drosophila. Univ. Texas Publ., 4228, 23-52.

zOUROS, E., LOFDAHL, K. AND MARTIN, P. A. 1988. Male hybrid sterility in Drosophila: Interactions between autosomes and sex chromosomes in crosses of $D$. mojavensis and $D$. arizonensis. Evolution, 42, 1321-1331. 